\title{
Biomarker correlation network in colorectal carcinoma by tumor anatomic location
}

\author{
Reiko Nishihara ${ }^{1,2,3,4^{*}}$ D, Kimberly Glass ${ }^{5}$, Kosuke Mima ${ }^{6}$, Tsuyoshi Hamada ${ }^{6}$, Jonathan A. Nowak , Zhi Rong Qian ${ }^{6}$, \\ Peter Kraft ${ }^{4}$, Edward L. Giovannucci ${ }^{4,5}$, Charles S. Fuchs ${ }^{5,6,7,8,9}$, Andrew T. Chan ${ }^{5,10}$, John Quackenbush ${ }^{3,11}$, \\ Shuji Ogino ${ }^{1,4,6,12^{*+}}$ and Jukka-Pekka Onnela ${ }^{3^{*}+}$
}

\begin{abstract}
Background: Colorectal carcinoma evolves through a multitude of molecular events including somatic mutations, epigenetic alterations, and aberrant protein expression, influenced by host immune reactions. One way to interrogate the complex carcinogenic process and interactions between aberrant events is to model a biomarker correlation network. Such a network analysis integrates multidimensional tumor biomarker data to identify key molecular events and pathways that are central to an underlying biological process. Due to embryological, physiological, and microbial differences, proximal and distal colorectal cancers have distinct sets of molecular pathological signatures. Given these differences, we hypothesized that a biomarker correlation network might vary by tumor location.
\end{abstract}

Results: We performed network analyses of 54 biomarkers, including major mutational events, microsatellite instability (MSI), epigenetic features, protein expression status, and immune reactions using data from 1380 colorectal cancer cases: 690 cases with proximal colon cancer and 690 cases with distal colorectal cancer matched by age and sex. Edges were defined by statistically significant correlations between biomarkers using Spearman correlation analyses. We found that the proximal colon cancer network formed a denser network (total number of edges, $n=173$ ) than the distal colorectal cancer network $(n=95)(P<0.0001$ in permutation tests). The value of the average clustering coefficient was 0.50 in the proximal colon cancer network and 0.30 in the distal colorectal cancer network, indicating the greater clustering tendency of the proximal colon cancer network. In particular, MSI was a key hub, highly connected with other biomarkers in proximal colon cancer, but not in distal colorectal cancer. Among patients with non-MSI-high cancer, BRAF mutation status emerged as a distinct marker with higher connectivity in the network of proximal colon cancer, but not in distal colorectal cancer.

Conclusion: In proximal colon cancer, tumor biomarkers tended to be correlated with each other, and MSI and BRAF mutation functioned as key molecular characteristics during the carcinogenesis. Our findings highlight the importance of considering multiple correlated pathways for therapeutic targets especially in proximal colon cancer.

\section{Background}

Colorectal cancer evolves through a progressive accumulation of genetic and epigenetic alterations that are influenced by the environment and host immunity. The interplay of molecular alterations forms biological interaction networks

\footnotetext{
*Correspondence: rnishiha@hsph.harvard.edu; shuji_ogino@dfci.harvard.edu; onnela@hsph.harvard.edu

${ }^{\dagger}$ Equal contributors

${ }^{1}$ Program of MPE Molecular Pathological Epidemiology, Department of Pathology, Brigham and Women's Hospital and Harvard Medical School, Boston, MA, USA

${ }^{3}$ Department of Biostatistics, Harvard T.H. Chan School of Public Health, Boston, MA, USA

Full list of author information is available at the end of the article
}

in colorectal cancer [1]. For example, epigenetic changes are known to be associated with genetic changes, as exemplified by the well-established link between $M L H 1$ promoter hypermethylation and microsatellite instability (MSI) [2, 3]. Additionally, microRNAs including MIR21 and MIR155 are reported to regulate gene expression [4-6], resulting in abnormal loss and overexpression of proteins [7]. In the tumor microenvironment, tumor-host interactions manifest as lymphocytic reactions directed at the tumor [8]. Given this multitude of molecular interactions, it is challenging to comprehensively understand the perturbations of the complex biological system and identify the key, underlying molecular events that drive colorectal 
carcinogenesis. Network analysis is recognized as an emerging approach to evaluating multidimensional tumor molecular data with the aim of revealing critical molecular events and pathways $[9,10]$.

Numerous lines of evidence indicate differences between proximal colon cancer, and distal colon and rectal cancer [11-13]. When compared with distal colon and rectal cancer, proximal colon cancer exhibits a higher prevalence of microsatellite instability (MSI) and highlevel $\mathrm{CpG}$ island methylator phenotype (CIMP-high) [11]. The proximal colon and distal colorectum have different embryologic origins; the former is derived from the midgut with blood supply from superior mesenteric artery, while the latter is derived from the hindgut with inferior mesenteric artery. Moreover, epithelial cells in the proximal colon are exposed to different bowel contents, including microbiota, compared with the distal colon and rectum [13]. Based on these differences in molecular alterations and tumor-host interactions with the microenvironment, the underlying etiology may differ between proximal and distal colorectal cancer. Accordingly, the structure of the biomarker correlation network may differ by tumor location. A better understanding of the connectivity and correlation of molecular events in biomarker networks can provide new insights into colorectal cancer etiology and pathogenesis, potentially advancing prevention and treatment strategies. In cancer biological networks, highly connected molecular markers, called hubs, play essential roles in carcinogenesis $[14,15]$. We hypothesized that correlation network structures and hub biomarkers might differ between proximal and distal colorectal cancer. Utilizing colorectal cancer databases of the Nurses' Health Study (NHS) and the Health Professionals Follow-up Study (HPFS), we examined the status of key driver mutations, MSI, CIMP, expression of selected proteins in tumor cells, and immune reactions to colorectal cancer.

\section{Results}

Comparison of biomarker networks by tumor location Within the NHS and the HPFS, we identified 1591 colorectal cancer patients with analyzed molecular features of colorectal tumors (Table 1). Biomarkers in this analysis included somatic oncogenic mutations, epigenetic features, protein expression levels, and host immune reactions in colorectal carcinoma. We conducted network analyses stratified by tumor location in a subset $(n=1380)$ of these patients. This subset included 690 patients with proximal colon cancer and 690 patients with distal colorectal cancer, matched by age and sex. Biomarker availability differed by tumor location only for the following three biomarkers: the intratumoral periglandular reaction (95.9\% available in proximal colon, $91.6 \%$ in distal colorectum), peritumoral lymphocytic reaction $(95.8 \%$ in proximal colon, $91.2 \%$ in distal colorectum), and tumor infiltrating lymphocytes (TIL) $(95.9 \%$ in proximal colon, $91.6 \%$ in distal colorectum), with the significance level of $P=0.05$ after the Bonferroni correction. The demographic characteristics of patients according to tumor location are described in Table 2. Patients with proximal colon cancer tended to have tumors with higher TNM stage and poor differentiation compared with patients with distal colorectal cancer.

In the correlation network analysis, a node represented a tumor tissue biomarker, and an edge was defined as a correlation between two nodes based on Spearman correlation analysis. Compared with the distal colorectal cancer network, the proximal colon cancer network had more edges and greater median degree (Table 3, Fig. 1). Nodes in the proximal colon cancer network tended to have a higher degree than those in the distal colorectal cancer network $(P=0.043$ based on a K-S test, Fig. 2). The median degree value was 3.0 in the proximal colon cancer network and 2.0 in the distal colorectal cancer network. The total number of edges was significantly greater in the proximal colon cancer network $(n=173)$ than in the distal colorectal cancer network $(n=95)$ in a permutation test where tumor location variable was permuted among 1380 patients $(P<0.0001)$. Also, we observed significant difference in the total number of edges in another permutation test where values of each biomarker were permuted within proximal colon cancer patients and distal colorectal cancer patients separately $(P<0.0001)$. In addition, the value of the average clustering coefficient indicated that the nodes in the proximal colon cancer network tended to cluster together more than those in the distal colorectal cancer network (Table 3). We defined hubs as nodes with a high degree centrality (high connectivity with other nodes) based on the overall colorectal cancer network that pooled both proximal colon and distal colorectal cancer. Nodes with degree centrality greater than the 80th percentile were considered as hubs. We found hubs in the proximal colon cancer network, including MSI and MLH1 methylation, but not in the distal colorectal cancer network (Table 3). For these biomarkers, degree centrality was computed as the fraction of nodes to which a node was connected. The values of degree centrality in the proximal colon cancer network and the distal colorectal cancer network were 0.39 and 0.17 for MSI, 0.36 and 0.21 for $M L H 1$ methylation. Within the distal colorectal cancer network, most of the nodes have a small number of connections. Methylation-related markers tended to have high degree centrality, although these markers were not hubs. The degree centrality values were 0.21 for $M L H 1$ methylation, 0.19 for methylations in CACNA1G, NEUROG1, RUNX3, and SOCS1and 0.19 for $B R A F$ mutation, and 0.19 for TIL. 
Table 1 Tumor molecular and pathological features in colorectal carcinomas

\begin{tabular}{|c|c|c|c|c|c|}
\hline Biomarker & Status measured & $\begin{array}{l}\% \text { or mean }(S D) \text { of the status } \\
\text { in proximal colon }(n=690)^{a}\end{array}$ & $\begin{array}{l}\text { \% or mean (SD) of the } \\
\text { status in distal colorectum } \\
(n=690)^{\mathrm{a}}\end{array}$ & $\begin{array}{l}\% \text { availability in } \\
\text { proximal colon } \\
(n=690)\end{array}$ & $\begin{array}{l}\% \text { availability in distal } \\
\text { colorectum }(n=690)\end{array}$ \\
\hline \multicolumn{6}{|c|}{ Somatic oncogenic mutations } \\
\hline BRAF & Mutation & $25.1 \%$ & $5.6 \%$ & $86.1 \%$ & $87.4 \%$ \\
\hline KRAS & Mutation & $43.0 \%$ & $36.6 \%$ & $86.2 \%$ & $87.5 \%$ \\
\hline PIK3CA & Mutation & $18.4 \%$ & $14.5 \%$ & $79.7 \%$ & $80.9 \%$ \\
\hline \multicolumn{6}{|l|}{ Methylation status } \\
\hline MLH1 & Methylation & $25.3 \%$ & $3.9 \%$ & $83.5 \%$ & $82.5 \%$ \\
\hline CDKN2A & Methylation & $44.3 \%$ & $21.8 \%$ & $83.5 \%$ & $82.5 \%$ \\
\hline CACNAIG & Methylation & $40.3 \%$ & $9.9 \%$ & $83.5 \%$ & $82.3 \%$ \\
\hline CRABP1 & Methylation & $52.8 \%$ & $20.9 \%$ & $83.5 \%$ & $82.5 \%$ \\
\hline IGF2 & Methylation & $44.3 \%$ & $12.1 \%$ & $83.5 \%$ & $82.5 \%$ \\
\hline NEUROG1 & Methylation & $53.1 \%$ & $21.0 \%$ & $83.5 \%$ & $82.3 \%$ \\
\hline RUNX3 & Methylation & $40.1 \%$ & $8.8 \%$ & $83.5 \%$ & $82.5 \%$ \\
\hline SOCS1 & Methylation & $30.4 \%$ & $7.9 \%$ & $83.3 \%$ & $82.5 \%$ \\
\hline LINE-1 & $\begin{array}{l}\text { Degree of } \\
\text { methylation (\%) }\end{array}$ & $64.7(S D, 9.7)$ & $62.6(S D, 9.9)$ & $85.1 \%$ & $84.5 \%$ \\
\hline IGF2 DMRO & Methylation & $22.0 \%$ & $29.6 \%$ & $65.1 \%$ & $65.2 \%$ \\
\hline \multicolumn{6}{|c|}{ Microsatellite instability (MSI) } \\
\hline MSI & MSI-high & $28.2 \%$ & $4.9 \%$ & $85.9 \%$ & $85.7 \%$ \\
\hline \multicolumn{6}{|l|}{ Protein expression } \\
\hline $\mathrm{CDH} 1$ & Loss & $52.6 \%$ & $50.9 \%$ & $44.9 \%$ & $42.5 \%$ \\
\hline CDKN1A & Loss & $72.3 \%$ & $89.2 \%$ & $52.8 \%$ & $55.2 \%$ \\
\hline CDKN1B & Loss & $47.9 \%$ & $34.8 \%$ & $51.7 \%$ & $53.8 \%$ \\
\hline CDKN2A & Loss & $35.3 \%$ & $17.9 \%$ & $46.8 \%$ & $47.8 \%$ \\
\hline CDX2 & Loss & $36.6 \%$ & $17.2 \%$ & $43.2 \%$ & $39.7 \%$ \\
\hline MGMT & Loss & $34.7 \%$ & $38.9 \%$ & $42.6 \%$ & $43.9 \%$ \\
\hline AURKA & Overexpression & $22.2 \%$ & $14.3 \%$ & $32.6 \%$ & $28.4 \%$ \\
\hline CCND1 & Overexpression & $82.6 \%$ & $73.5 \%$ & $64.9 \%$ & $65.5 \%$ \\
\hline CD274 & Overexpression & $85.6 \%$ & $83.7 \%$ & $43.2 \%$ & $40.0 \%$ \\
\hline CDK8 & Overexpression & $72.6 \%$ & $69.4 \%$ & $30.7 \%$ & $26.5 \%$ \\
\hline CTNNB1 (nuclear) & Overexpression & $32.7 \%$ & $58.2 \%$ & $75.8 \%$ & $74.2 \%$ \\
\hline CTSB & Overexpression & $82.7 \%$ & $82.9 \%$ & $45.2 \%$ & $42.3 \%$ \\
\hline DNMT3B & Overexpression & $19.7 \%$ & $10.3 \%$ & $45.5 \%$ & $49.3 \%$ \\
\hline EPAS1 & Overexpression & $47.0 \%$ & $44.5 \%$ & $46.2 \%$ & $42.0 \%$ \\
\hline FASN & Overexpression & $61.1 \%$ & $60.0 \%$ & $64.8 \%$ & $65.9 \%$ \\
\hline HGF & Overexpression & $45.8 \%$ & $47.9 \%$ & $39.6 \%$ & $37.2 \%$ \\
\hline HIF1A & Overexpression & $17.0 \%$ & $20.1 \%$ & $47.7 \%$ & $42.6 \%$ \\
\hline IGF2BP3 & Overexpression & $36.8 \%$ & $32.7 \%$ & $43.8 \%$ & $41.2 \%$ \\
\hline IRS1 & Overexpression & $29.5 \%$ & $30.6 \%$ & $44.6 \%$ & $41.7 \%$ \\
\hline IRS2 & Overexpression & $31.6 \%$ & $33.2 \%$ & $44.9 \%$ & $41.9 \%$ \\
\hline PPARG & Overexpression & $24.1 \%$ & $19.2 \%$ & $33.6 \%$ & $29.4 \%$ \\
\hline PTGER2 & Overexpression & $27.8 \%$ & $26.3 \%$ & $45.8 \%$ & $41.3 \%$ \\
\hline PTGS2 & Overexpression & $53.5 \%$ & $69.7 \%$ & $81.7 \%$ & $79.9 \%$ \\
\hline SIRT1 & Overexpression & $38.6 \%$ & $37.9 \%$ & $39.4 \%$ & $35.9 \%$ \\
\hline
\end{tabular}


Table 1 Tumor molecular and pathological features in colorectal carcinomas (Continued)

\begin{tabular}{|c|c|c|c|c|c|}
\hline STAT3 & Overexpression & $54.7 \%$ & $54.0 \%$ & $46.4 \%$ & $43.2 \%$ \\
\hline TP53 & Overexpression & $33.0 \%$ & $53.2 \%$ & $66.7 \%$ & $67.2 \%$ \\
\hline VDR & Overexpression & $37.0 \%$ & $38.2 \%$ & $47.4 \%$ & $42.5 \%$ \\
\hline YAP1 (cytoplasmic) & Overexpression & $21.3 \%$ & $17.7 \%$ & $44.2 \%$ & $41.0 \%$ \\
\hline JC Virus T-Antigen (JCVT) & Overexpression & $28.4 \%$ & $40.7 \%$ & $43.9 \%$ & $47.7 \%$ \\
\hline \multicolumn{6}{|l|}{ Immune reactions } \\
\hline $\begin{array}{l}\text { Peritumoral lymphocytic } \\
\text { reaction }\end{array}$ & Greater reaction & $19.4 \%$ & $12.9 \%$ & $95.8 \%$ & $91.2 \%$ \\
\hline $\begin{array}{l}\text { Intratumoral periglandular } \\
\text { reaction }\end{array}$ & Greater reaction & $17.2 \%$ & $9.0 \%$ & $95.9 \%$ & $91.6 \%$ \\
\hline $\begin{array}{l}\text { Tumor infiltrating } \\
\text { lymphocytes (TIL) }\end{array}$ & Greater reaction & $16.3 \%$ & $4.4 \%$ & $95.9 \%$ & $91.6 \%$ \\
\hline Crohn's-like reaction & Greater reaction & $9.9 \%$ & $5.0 \%$ & $80.4 \%$ & $73.2 \%$ \\
\hline CD3+ in cancer area & Density (cells/mm²) & $716.6(S D, 1501.8)$ & $696.5(S D, 1560.7)$ & $47.1 \%$ & $42.9 \%$ \\
\hline CD8+ in cancer area & Density (cells/mm²) & $814.7(S D, 1741.0)$ & $700.9(S D, 1582.1)$ & $46.7 \%$ & $41.7 \%$ \\
\hline $\mathrm{CD} 45 \mathrm{RO}+$ in cancer area & Density (cells/mm²) & $711.4(S D, 1113.4)$ & $634.4(S D, 1244.7)$ & $48.1 \%$ & $43.6 \%$ \\
\hline FOXP3+ in cancer area & Density (cells/mm²) & $40.0(S D, 47.0)$ & $35.2(S D, 37.9)$ & $45.2 \%$ & $41.9 \%$ \\
\hline \multicolumn{6}{|l|}{ miRNA expression } \\
\hline MIR21 & $\begin{array}{l}\text { Normalized } \\
\text { expression level }\end{array}$ & $8.3(S D, 13.0)$ & $5.6(S D, 4.0)$ & $53.2 \%$ & $48.6 \%$ \\
\hline MIR155 & $\begin{array}{l}\text { Normalized } \\
\text { expression level }\end{array}$ & $0.01(S D, 0.01)$ & $0.005(S D, 0.006)$ & $53.2 \%$ & $48.6 \%$ \\
\hline \multicolumn{6}{|l|}{ Microorganism } \\
\hline Fusobacterium nucleatum & Presence & $16.0 \%$ & $9.1 \%$ & $71.7 \%$ & $67.1 \%$ \\
\hline
\end{tabular}

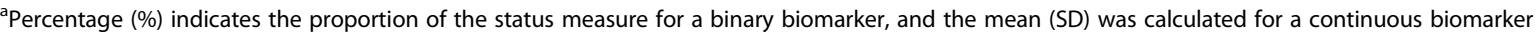
SD standard deviation

To identify biomarkers that had higher connectivity particularly in the proximal colon cancer network compared with the distal colorectal cancer network, we calculated Cook's distance (see Methods) for each highly-connected marker (those at or above the 80th percentile of the degree distribution in the overall colorectal cancer network) (Additional file 1: Figure S1). In Table 4, MSI showed the largest Cook's distance (0.12) and was highly connected with other markers in the proximal colon cancer network (degree $=21$ ), but not in the distal colorectal cancer network (degree $=9$ ). In the proximal colon cancer network, MSI was positively correlated with MLH1 methylation (correlation coefficient $[\rho]=0.77$ ), CACNA1G methylation $(\rho=0.55)$, RUNX3 methylation $(\rho=0.50)$, SOCS1 methylation $(\rho=0.49)$, IGF2 methylation ( $\rho=0.46), C R A B P 1$ methylation $(\rho=0.45), B R A F$ mutation $(\rho=0.44)$, TIL $(\rho=0.41)$, NEUROG1 methylation $(\rho=0.37)$, Crohn's-like reaction $(\rho=0.37), C D K N 2 A$ methylation ( $\rho=0.33)$, abundance of Fusobacterium nucleatum ( $\rho=0.33)$, SIRT1 overexpression $(\rho=0.25)$, loss of CDX2 expression $(\rho=0.25)$, CCND1 overexpression $(\rho=0.25)$, LINE-1 methylation $(\rho=0.24)$, peritumoral lymphocytic reaction $(\rho=0.24)$, MIR155 expression $(\rho=0.22)$, and intratumoral periglandular reaction
( $\rho=0.22)$. MSI was negatively correlated with KRAS mutation $(\rho=-0.39)$ and loss of CDKN1A expression ( $\rho=-0.36$ ), suggesting that these molecular events tended to be mutually exclusive with MSI-high. In the distal colorectal cancer network, MSI was also positively correlated with $M L H 1$ methylation $(\rho=0.35)$, TIL ( $\rho=0.29)$, RUNX3 methylation $(\rho=0.26)$, Crohn'slike reaction $(\rho=0.24)$, CACNA1G methylation $(\rho=0.23)$, SOCS1 methylation $(\rho=0.22)$, intratumoral periglandular reaction $(\rho=0.22), B R A F$ mutation $(\rho=0.21)$, and peritumoral lymphocytic reaction $(\rho=0.19)$. These were the subset of biomarkers positively correlated with MSI in the proximal colon cancer network, implying a partly shared mechanism by which MSI promotes perturbation of cellular and molecular functions. Moreover, the methylation markers of $M L H 1, I G F 2$, and CACNA1G had a higher degree in the proximal colon cancer network compared with the distal colorectal cancer network. As shown in Fig. 1, negative correlations were observed between KRAS mutation and other biomarkers in the proximal colon cancer network, but not in the distal colorectal cancer network. In the proximal colon cancer network, KRAS mutation was negatively correlated with $B R A F$ mutation $(\rho=-0.47)$, MLH1 methylation $(\rho=-0.40)$, MSI-high 
Table 2 Demographic, clinical and pathologic features of colorectal cancers in the network analysis dataset by tumor location

\begin{tabular}{|c|c|c|c|c|}
\hline & Total $(n=1380)$ & Proximal colon cancer $(n=690)$ & Distal colorectal cancer $(n=690)$ & $P$ value $^{\mathrm{a}}$ \\
\hline Age, mean (SD) & $69.9(8.6)$ & $70.0(8.8)$ & $69.7(8.5)$ & 0.56 \\
\hline \multicolumn{5}{|l|}{ Sex } \\
\hline Female & $758(54.9 \%)$ & $379(54.9 \%)$ & $379(54.9 \%)$ & \multirow[t]{2}{*}{0.99} \\
\hline Male & $622(45.1 \%)$ & $311(45.1 \%)$ & $311(45.1 \%)$ & \\
\hline \multicolumn{5}{|l|}{ Tumor location } \\
\hline Cecum & $253(18.3 \%)$ & $253(36.7 \%)$ & $0(0 \%)$ & \multirow[t]{9}{*}{$<0.0001$} \\
\hline Ascending colon & $303(22.0 \%)$ & $303(43.9 \%)$ & $0(0 \%)$ & \\
\hline Hepatic flexure & $45(3.3 \%)$ & $45(6.5 \%)$ & $0(0 \%)$ & \\
\hline Transverse colon & $89(6.4 \%)$ & 89 (12.9\%) & $0(0 \%)$ & \\
\hline Splenic flexure & $31(2.2 \%)$ & $0(0 \%)$ & $31(4.5 \%)$ & \\
\hline Descending colon & $66(4.8 \%)$ & $0(0 \%)$ & $66(9.6 \%)$ & \\
\hline Sigmoid colon & $296(21.4 \%)$ & $0(0 \%)$ & $296(42.9 \%)$ & \\
\hline Rectosigmoid junction & $100(7.2 \%)$ & $0(0 \%)$ & $100(14.5 \%)$ & \\
\hline Rectum & $197(14.3 \%)$ & $0(0 \%)$ & $197(28.6 \%)$ & \\
\hline \multicolumn{5}{|l|}{ TNM Stage } \\
\hline । & $324(26.2 \%)$ & $146(22.9 \%)$ & $178(29.7 \%)$ & \multirow[t]{4}{*}{0.002} \\
\hline$\|$ & $404(32.7 \%)$ & $232(36.4 \%)$ & $172(28.7 \%)$ & \\
\hline III & $332(26.8 \%)$ & $159(24.9 \%)$ & $173(28.9 \%)$ & \\
\hline IV & $177(14.3 \%)$ & $101(15.8 \%)$ & $76(12.7 \%)$ & \\
\hline \multicolumn{5}{|l|}{ Tumor differentiation } \\
\hline Well to moderate & $1231(89.2 \%)$ & $577(83.6 \%)$ & $654(94.8 \%)$ & \multirow[t]{2}{*}{$<0.0001$} \\
\hline Poor & $149(10.8 \%)$ & $113(16.4 \%)$ & $36(5.2 \%)$ & \\
\hline \multicolumn{5}{|c|}{ Family history of colorectal cancer in first-degree relative(s) } \\
\hline No & $1074(78.4 \%)$ & $532(77.9 \%)$ & $542(78.9 \%)$ & \multirow[t]{2}{*}{0.65} \\
\hline Yes & $296(21.6 \%)$ & $151(22.1 \%)$ & $145(21.1 \%)$ & \\
\hline
\end{tabular}

The \% numbers indicate the fraction of cases with a given feature among total cases, proximal colon cancer cases, or distal colorectal cancer cases

${ }^{a} P$ value was calculated using a t-test for age and chi-squared tests for categorical variables

( $\rho=-0.39)$, RUNX3 methylation $(\rho=-0.34), C A C-$ NA1G methylation $(\rho=-0.32), C R A B P 1$ methylation $(\rho=-0.31)$, IGF2 methylation $(\rho=-0.30)$, loss of CDX2 expression $(\rho=-0.29)$, SOCS1 methylation $(\rho=-0.27), C D K N 2 A$ methylation $(\rho=-0.23)$, NEUROG1 methylation $(\rho=-0.22)$, and Crohn's-like reaction $(\rho=-0.20)$.

\section{Biomarker networks constructed in non-MSI-high colorectal cancer}

The higher frequency of MSI-high in the proximal colon cancer network compared with the distal colorectal cancer network could potentially result in the higher connectivity of the former. Therefore, as a secondary analysis, we restricted our analysis to patients with non-MSI-high cancer, and constructed networks by tumor location. After matching by age and sex, there were 246 patients with non-MSI-high proximal colon cancer and 246 patients with non-MSI-high distal colorectal cancer (Additional file 2: Figure S2). Compared with the distal colorectal cancer network, we observed a slightly higher connectivity and more hubs in the proximal colon cancer network. The total numbers of edges was 64 in the proximal colon cancer network and 56 in the distal colorectal cancer network (Table 3). Highly connected biomarkers (with degree centrality) were $B R A F$ mutation (0.19) and methylation-related markers including CDKN2A (0.17), IGF2 (0.17), RUNX3 (0.17), CACNA1G (0.15), CRABP1 (0.15), and NEUROG1 $(0.15)$ in the proximal colon cancer network. In the distal colorectal cancer network, two biomarkers were hubs including NEUROG1 methylation (degree centrality $=0.15$ ) and RUNX3 methylation (degree centrality $=0.15$ ). Among non-MSI-high cases, BRAF mutation was the most distinct marker that was strongly correlated with other markers in the proximal cancer network (degree $=10)$, but not in the distal cancer network (degree $=3$ ) (Cook's distance $=0.17$ ). In the proximal colon cancer network, KRAS mutation tended to be mutually exclusive only with $B R A F$ mutation, indicating that the observed 
Table 3 Network characteristics by tumor location

\begin{tabular}{|c|c|c|c|}
\hline & Total $(N=1380)$ & Proximal colon cancer $(N=690)$ & Distal colorectal cancer $(N=690)$ \\
\hline \multicolumn{4}{|c|}{ Correlation network based on original variables } \\
\hline Number of nodes & 54 & 54 & 54 \\
\hline Number of edges & 268 & 173 & 95 \\
\hline Median degree & 8.0 & 3.0 & 2.0 \\
\hline Average clustering coefficient & 0.52 & 0.50 & 0.30 \\
\hline Hubs $^{a}$ (degree centrality) & $\begin{array}{l}\text { RUNX3 (0.47) } \\
\text { CACNA1G (0.45) } \\
\text { MSI }(0.45) \\
\text { MLH1 (0.43) } \\
\text { TIL }(0.43) \\
\text { BRAF }(0.40) \\
\text { NEUROG1 }(0.40) \\
\text { CRABP1 }(0.38) \\
\text { IGF2 }(0.38) \\
\text { SOCS1 }(0.38) \\
\text { CDKN2A }(0.36) \\
\text { MIR155 }(0.36)\end{array}$ & $\begin{array}{l}\text { MSI (0.39) } \\
\text { MLH1 (0.36) }\end{array}$ & - \\
\hline \multicolumn{4}{|c|}{ Correlation network in non-MSI-high cancer } \\
\hline Number of nodes & 53 & 53 & 53 \\
\hline Median degree & 4.0 & 1.0 & 1.0 \\
\hline Number of edges & 120 & 64 & 56 \\
\hline Average clustering coefficient & 0.33 & 0.23 & 0.26 \\
\hline Hubs $^{a}$ (degree centrality) & $\begin{array}{l}\text { RUNX3 (0.15) } \\
\text { NEUROG1 (0.15) }\end{array}$ & $\begin{array}{l}\text { BRAF }(0.19) \\
\text { CDKN2A }(0.17) \\
\text { IGF2 }(0.17) \\
\text { RUNX3 }(0.17) \\
\text { CACNA1G }(0.15) \\
\text { CRABP1 }(0.15) \\
\text { NEUROG1 }(0.15)\end{array}$ & $\begin{array}{l}\text { RUNX3 (0.15) } \\
\text { NEUROG1 (0.15) }\end{array}$ \\
\hline \multicolumn{4}{|c|}{ Correlation network based on the same number of edges } \\
\hline Number of nodes & 54 & 54 & 54 \\
\hline Median degree & 1.5 & 4.0 & 1.0 \\
\hline Number of edges & 100 & 100 & 100 \\
\hline Average clustering coefficient & 0.27 & 0.29 & 0.27 \\
\hline Hubs $^{a}$ (degree centrality) & $\begin{array}{l}\text { CACNA1G (0.26) } \\
\text { IGF2 }(0.26) \\
\text { RUNX3 }(0.25) \\
\text { BRAF }(0.23) \\
\text { MLH1 }(0.23) \\
\text { MSI }(0.23) \\
\text { CRABP1 }(0.21) \\
\text { TIL }(0.21) \\
\text { CDKN2A }(0.19) \\
\text { NEUROG1 }(0.19) \\
\text { SOCS1 }(0.19)\end{array}$ & $\begin{array}{l}\text { CACNA1G (0.28) } \\
\text { MLH1 }(0.26) \\
\text { MSI }(0.26) \\
\text { IGF2 }(0.25) \\
\text { RUNX3 }(0.25) \\
\text { BRAF }(0.23) \\
\text { CRABP1 (0.23) } \\
\text { CDKN2A }(0.21) \\
\text { TIL }(0.21) \\
\text { NEUROG1 }(0.19) \\
\text { SOCS1 }(0.17)\end{array}$ & $\begin{array}{l}\text { RUNX3 (0.19) } \\
\text { IGF2 (0.17) } \\
\text { NEUROG1 (0.17) }\end{array}$ \\
\hline
\end{tabular}

${ }^{a}$ Markers with degree centrality at or above the 80th percentile in the colorectal cancer network ${ }^{\mathrm{b}} \mathrm{CDKN2A}$ promoter hypermethylation

MSI microsatellite instability, TIL Tumor infiltrating lymphocytes

negative correlations of KRAS mutation with methylationrelated markers and other biomarkers might be confounded by MSI-high in our earlier analysis.

\section{Biomarker networks with equal edge counts}

To examine whether the difference in the number of edges across the two networks affected hub identification (designation of nodes as hubs), we modified each network by retaining 100 of the largest Spearman correlation coefficients in absolute values regardless of their $P$-values, resulting in two networks with 100 edges (Additional file 3: Figure S3). The median degree was 4.0 in the proximal colon cancer network and 1.0 in the distal colorectal cancer network, indicating that edges were confined to 

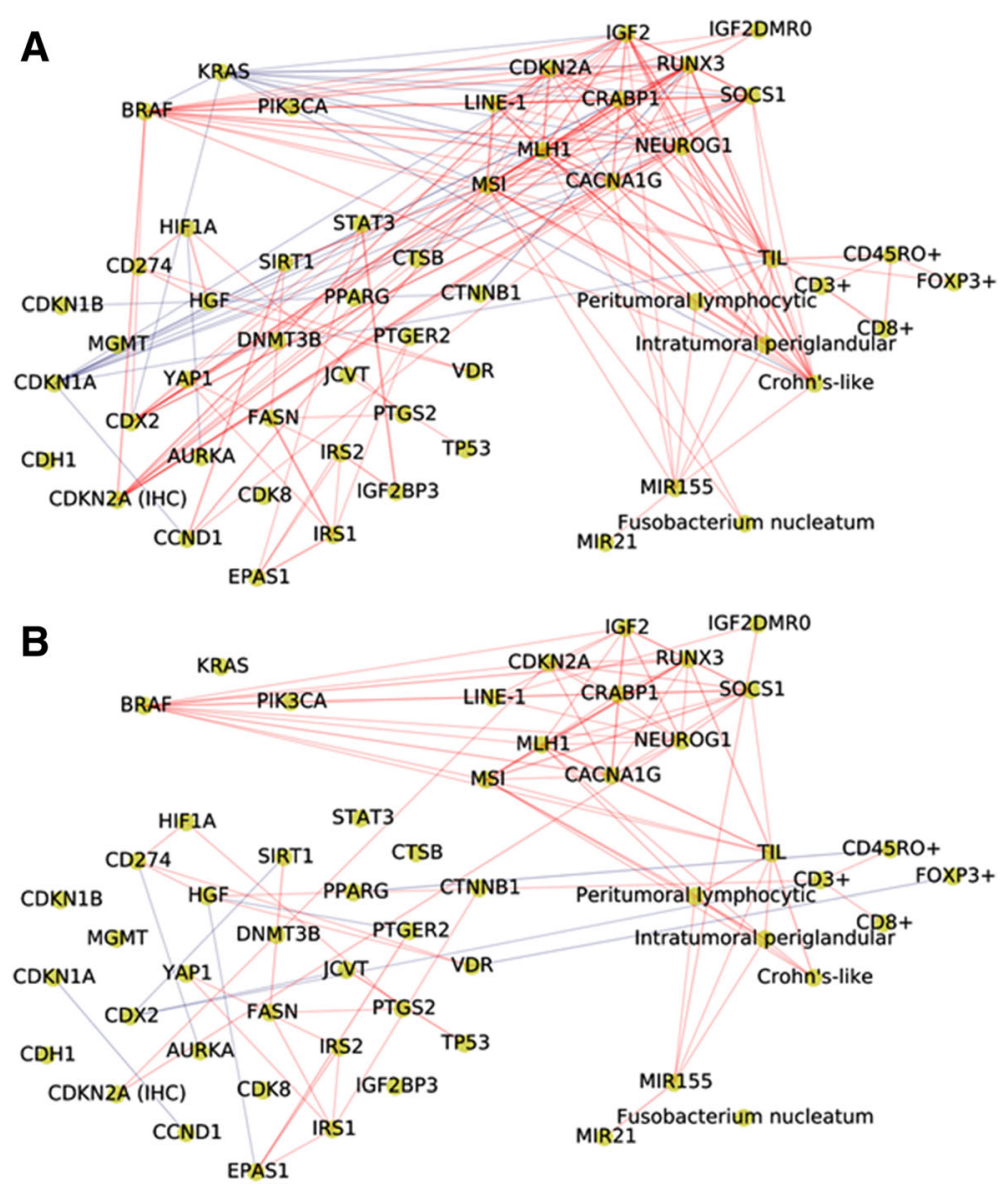

Fig. 1 Biomarker networks by tumor location; proximal colon cancer network (a), and distal colorectal cancer network (b). A node represents a molecular feature, and an edge specifies a statistically significant Spearman correlation between two markers (nodes) with a significance level of $3.5 \times 10^{-5}(0.05 / 1431$, based on the Bonferroni correction). The red line indicates a positive correlation, and the blue line indicates a negative correlation; line width is proportional to correlation coefficient. CDKN2A (IHC), protein expression of CDKN2A; CDKN2A, methylation level of CDKN2A; LINE-1, methylation level of long interspersed nucleotide element 1; MSI, microsatellite instability; TIL, lymphocytes on top of neoplastic epithelial cells

particular biomarkers in the proximal colon cancer network. When defined as markers with degree centrality at or above the 80th percentile in the colorectal cancer network, there were 11 hubs including methylation-related markers, MSI, BRAF mutation, and TIL in the proximal colon cancer network (Table 3). In contrast, only three methylation-related markers were identified as hubs in the distal colorectal cancer network. This finding supports the characteristics of the proximal colon cancer network observed in the original network analysis, where MSI and methylation-related markers were associated with perturbation of other molecular events.

\section{Sensitivity analyses}

Across different significance levels in the Spearman correlation analyses, the total number of edges in the proximal colon cancer network was consistently greater than in the distal colorectal cancer network, supporting a more highly correlated biomarker network in proximal colon cancer (Fig. 3). In addition, we constructed biomarker networks based on the Spearman correlation analyses using binary variables for all markers and still found that the proximal colon cancer network was denser than the distal colorectal cancer network (Additional file 4: Table S1). Moreover, the proximal colon cancer remained denser when the analysis included only biomarkers with missing data in less than $20 \%$ of the patients (Additional file 4: Table S1).

\section{Discussion}

Within a tumor molecular dataset including major mutational events, MSI, epigenetic features, protein expression status, and host immune reactions in 1380 colorectal cancer patients, we conducted a network analysis to evaluate biomarker network structure in proximal colon cancer and distal colorectal cancer. We found that 


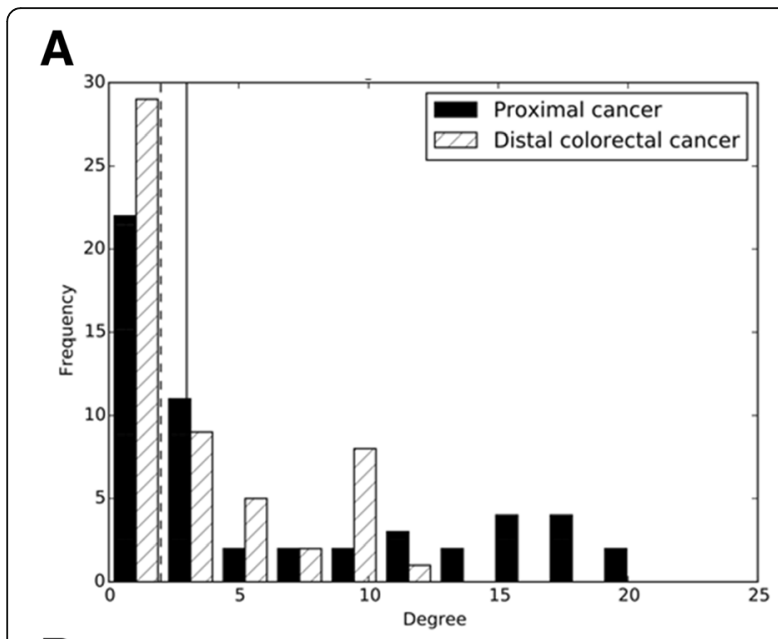

B

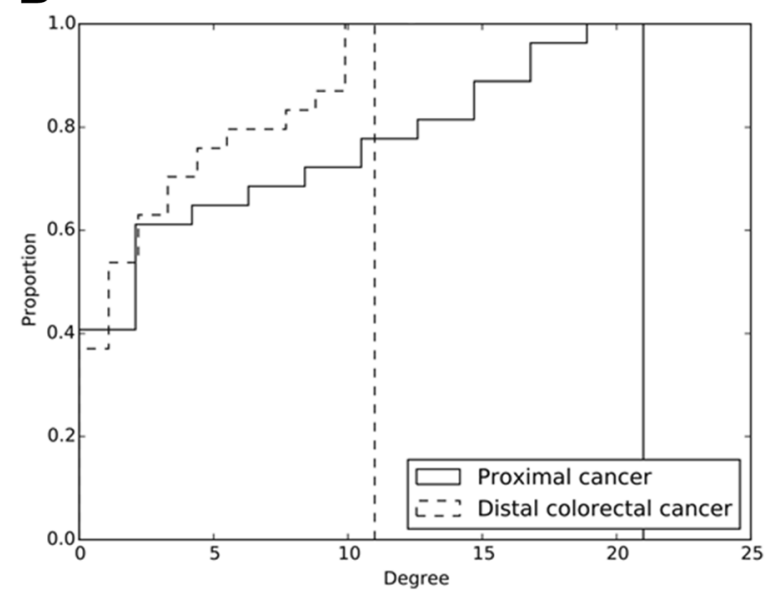

Fig. 2 a Degree distribution in biomarker networks by tumor location. b Cumulative degree distribution in biomarker networks by tumor location. The solid (the distal colorectal cancer network) and dashed lines (the proximal colon cancer network) indicate the median degree values

in proximal colon cancer there were many highly correlated biomarkers, leading to an overall denser network as compared with distal colorectal cancer. In the distal colorectal cancer network, biomarkers had fewer connections and were less clustered, resulting in fewer hubs. This finding indicates that carcinogenic events in distal colorectal cancer tended to occur independently from each other. In the proximal colon cancer network, MSI was a hub and had differential connectivity with other biomarkers compared with the distal colorectal cancer network. In both the proximal colon and distal colorectal cancer networks, we observed common biomarkers that positively correlated with MSI including methylation-related markers, $B R A F$ mutation, and immune reactions, while overexpression or loss of protein expression associated with MSI were further found only in the proximal colon cancer network. In addition to MSI, CpG island methylation in $M L H 1, I G F 2$, and CACNA1G genes were highly connected to other biomarkers mainly in proximal colon cancer. Among non-MSI-high patients, BRAF mutation status was the most distinct marker that was strongly associated with other molecular events in the proximal colon cancer network, but not in the distal colorectal cancer network. The sensitivity analyses consistently showed a denser network in proximal colon cancer when compared with the distal colorectal cancer network. Our results indicate that many molecular events occur in relation to MSI in proximal colon cancer, and that MSI and $B R A F$ mutation played important roles in the carcinogenic process of proximal colon cancer.

In colorectal cancer and other types of cancer, previous biomarker network studies have shown the importance of interdependence of mutation, methylation, and protein expression [16, 17]. In a biological network, hubs (highly connected nodes) are considered to be drivers playing an essential role during tumorigenesis [14, 15]. Previous studies showed that $M L H 1$ promoter methylation causes mismatch repair deficiency and MSI [2, 3]. In sporadic cancer, it is not well known whether MSI causes mutations in oncogenes and tumor-suppressor genes, and aberrant protein expressions. Earlier studies reported that genomic instability conferred by somatic mutational alterations further generates mutations in oncogenes or anti-oncogenes $[18,19]$. Our results suggest that the role of MSI differ by tumor anatomic location; in proximal colon cancer, MSI might lead to perturbations of many molecular events, and in distal colorectal cancer molecular events are more likely independent from MSI. Consistent with our findings, MSI and CIMP-high were highly correlated and occurred more frequently in proximal colon cancer than in distal colorectal cancer [11]. Methylation signatures

Table 4 Markers with differential connectivity by tumor location based on Cook's distance among highly-connected markers ${ }^{a}$

\begin{tabular}{lllll}
\hline Highly-connected markers $^{a}$ & Degree centrality & Degree in proximal colon cancer & Degree in distal colorectal cancer & Cook's distance \\
\hline MSI & 0.45 & 21 & 9 & 0.12 \\
IGF2 & 0.38 & 18 & 9 & 0.028 \\
CRABP1 & 0.38 & 16 & 8 & 0.015 \\
CACNA1G & 18 & 10 & 0.0071 \\
TIL & 0.45 & 18 & 10 & 0.0071 \\
\hline
\end{tabular}

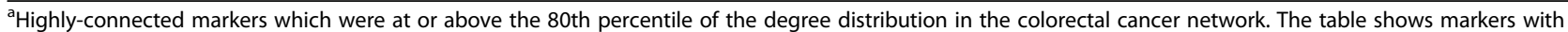
Cook's distance higher than 0.005

MSI microsatellite instability, TIL tumor infiltrating lymphocytes 


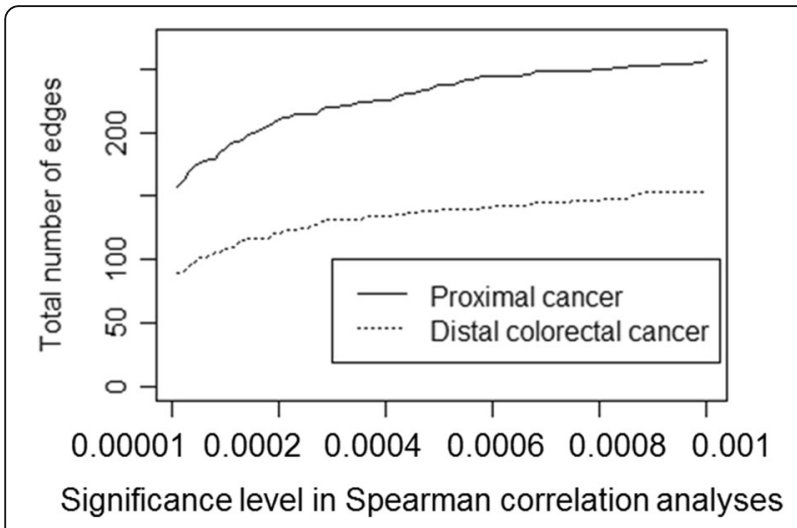

Fig. 3 Total number of edges in biomarker networks as a function of the significance levels in Spearman correlation analyses

measured in our study were CIMP-specific markers which were correlated with each other. Thus, higher frequencies of MSI and CIMP-high might contribute to the higher connectivity of the proximal colon cancer network. However, even when we excluded MSI cancer patients from the analyses, the higher connectivity persisted in the proximal colon cancer network. In addition, when we modified the two networks such that each had 100 edges, corresponding to the 100 most positive or negative correlation coefficients, the proximal colon cancer network remained to have more hubs and greater median degree than the distal colorectal cancer network. Our findings provide new insights into the carcinogenesis of proximal colon cancer, which possibly exhibits highly interactive biological mechanisms.

$B R A F$ oncogenic mutations are observed in $10 \%$ to $20 \%$ of colorectal cancer patients and are associated with MSI-high and CIMP-high phenotypes [19, 20]. The serine/threonine-protein kinase BRAF is involved in the mitogen-activated protein kinase (MAPK) pathway, which is associated with proliferation, cell growth, and differentiation [20]. In our study, CIMP-high was associated with $B R A F$ mutation in both proximal colon and distal colorectal cancer. Previous studies observed CIMP-high and $B R A F$ mutations in an early-stage colorectal neoplasm, and acquisition of $B R A F$ mutation was considered to be mediated by DNA hypermethylation of several genes, including IGFBP7 and $B M P 3$ [18]. The close relationship between CIMP and $B R A F$ mutation may drive carcinogenesis regardless of tumor location.

A large body of literature demonstrated that colorectal cancer patients with non-MSI-high and mutant $B R A F$ were associated with the highest mortality, whereas patients with MSI-high/BRAF-wild-type showed the lowest mortality when both MSI and $B R A F$ mutation status were considered [21-24]. Among proximal colon cancer patients, the non-MSI-high/BRAF-mutant subtype was associated with a worse prognosis [25]. Together with these previous studies, our findings suggest the critical role of $B R A F$ mutation in the absence of MSI-high especially in the proximal colon carcinogenesis.

Our results showed the strong negative correlation between $B R A F$ and $K R A S$ mutations specifically in proximal colon cancer. Both $B R A F$ and $K R A S$ oncogenes encode proteins involved in the MAPK pathway, and $B R A F$ mutation has been reported to be mutually exclusive with $K R A S$ mutation in colorectal cancer [26, 27]. $B R A F$ is more frequently mutated in the proximal colon than distal colorectum, while the frequency of KRAS mutation is more similar throughout the colorectum except for the cecum [11]. The biological correlation pattern between $B R A F$ and KRAS might depend on tumor location.

The distal colorectal cancer network was less densely connected than the proximal colon cancer network. Although we did not find biomarkers with high degree centrality, the promoter methylations, BRAF mutation, and TIL tended to be correlated with other molecular events. Distal carcinoma was previously reported to be more responsive to drug regimens, including 5fluorouracil-based chemotherapeutics and single-agent anti-epidermal growth factor receptor (EGFR) antibodies, while proximal carcinoma tended to show more resistance to those treatments $[12,26]$. Our results from the network analysis found that oncogenic processes are relatively independent from one another in distal colorectal cancer. Taken together, our data support a biological difference between colorectal carcinoma by tumor location.

We recognize that there are several limitations to our study. Although we chose widely recognized pathological biomarkers in colorectal cancer, our data did not cover all molecular pathological markers that have been reported in the literature, such as the mutation status of SMAD4 and PTEN [28, 29]. Our selection of the biomarkers might have resulted in a greater connectivity of the biomarker network in the proximal colon. Measurement errors could have existed in protein expression analyses using immunohistochemistry (IHC). Nonetheless, based on reviews by two independent pathologists, most of the IHC markers showed agreement levels generally considered to be reasonable in pathology research (kappa coefficient > 0.6). We conducted network analyses focusing on the proximal colon and distal colorectum, but not on more detailed segments (the cecum, ascending colon, hepatic flexure, transverse colon, splenic flexure, descending colon, sigmoid colon, rectosigmoid junction, and rectum). The importance of examining these segments warrants further investigations with even larger sample sizes. These results contribute to our understanding of colorectal carcinogenesis, suggesting a different mechanism by tumor location. Although our findings were corroborated by sensitivity analyses, future validation 
studies with independent data sets are necessary to confirm these findings.

Despite the limitations, our study has several strengths that distinguish it from previous studies. First, we measured different kinds of tumor biomarkers including somatic mutations, methylation levels, MSI status, immune reactions, and protein expressions. These measurements provided a reasonably comprehensive view of molecular interplay in the networks and enabled the identification of important carcinogenic pathways. Second, our overall sample size with 1380 cases enabled us to conduct robust network analyses using multiple biomarkers. Third, we collected colorectal tumors from hospitals and pathology laboratories across the U.S. (rather than one or a few hospitals), which increases generalizability of our findings.

\section{Conclusions}

Biomarkers in proximal colon cancer possessed higher connectivity while those in distal colorectal cancer tended to be independent from each other. In proximal colon cancer, MSI-high and BRAF mutation occurred in relation to many other tumor features, indicating their important roles during carcinogenesis. Our findings highlight the necessity of a systems therapeutic approach that can target both specific and multiple pathways of the proximal colon cancer network.

\section{Methods}

\section{Study population and design}

Initiated in 1976, the NHS enrolled 121,701 female nurses aged 30-55 years. Established in 1986, the HPFS enrolled 51,529 male health professionals including dentists, optometrists, osteopaths, pharmacists, podiatrists, and veterinarians aged $40-75$ years. Within these two cohorts, colorectal cancer patients were ascertained by biennial questionnaires, the National Death Index (for unreported lethal cases), and reviews of medical records. Study physicians reviewed medical and pathological records to retrieve detailed information on colorectal tumors including TNM stage, differentiation, and bowel subsites. For bowel subsite sections, the proximal colon included the cecum, ascending colon, hepatic flexure, and transverse colon; the distal colorectum included the splenic flexure, descending colon, sigmoid colon, rectosigmoid junction, and rectum.

We collected formalin-fixed paraffin-embedded (FFPE) archival tumor tissue blocks from pathology laboratories in U.S. hospitals where patients underwent tumor resection. A centralized pathological review of hematoxylin and eosin-stained tissue sections was conducted by a single pathologist (S.O.) for all colorectal carcinoma cases. Tumor differentiation was categorized into well to moderate or poor, based on the extent of glandular areas. Based on the availability of tumor tissue blocks, 1591 cases were selected for the molecular and pathological analyses. Patients with proximal colon cancer were matched by age and sex to patients with distal colorectal cancer by randomly selecting a patient diagnosed with proximal colon cancer from the same sex and age category $(<50,50-59$, $60-69$, or $\geq 70$ years). Subsequently, out of the 1591 patients in the original dataset, 1380 patients (690 patients with proximal colon cancer and 690 patients with distal colorectal cancer) were included in the network analysis dataset. For each marker, patients were excluded from an analysis when data on that marker were not available. Percentages of patients with available biomarkers are shown in Table 1.

\section{Molecular pathological analysis}

In the NHS and the HPFS, we utilized a colorectal cancer database which contained biomarker data on molecular alterations reported to play important roles in carcinogenic pathways in colorectal cancer. Tumor molecular and pathological markers in the database included mutational events, epigenetic features, protein expression levels, and host immune reactions in colorectal carcinoma. The analyses were performed as previously described. In FFPE tissues, DNA extraction and pyrosequencing were conducted for BRAF (codon 600), KRAS (codons 12, 13, 61, or 146), and PIK3CA (exons 9 and 20) [30-32]. The analysis of MSI was performed using 10 microsatellite markers (D2S123, D5S346, D17S250, BAT25, BAT26, BAT40, D18S55, D18S56, D18S67, and D18S487) [24]. We defined MSI-high as instability in $\geq 30 \%$ of the markers and MSI-low/microsatellite stability (MSS) as instability in $<30 \%$ of the markers [24]. To quantify the amount of Fusobacterium nucleatum DNA, quantitative polymerase chain reaction (PCR) assay was performed using the TaqMan primer/probe sets (Applied Biosystems) for the $n u s G$ gene of Fusobacterium nucleatum and for SLCO2A1 as a reference human gene [33]. Amplification and detection of DNA was performed with the StepOnePlus Real-Time PCR Systems (Applied Biosystems).

DNA methylation was quantified using validated bisulfite DNA treatment and real-time PCR (MethyLight) for eight $\mathrm{CpG}$ island specific promoters including $C A C N A 1 G$, CDKN2A (p16), CRABP1, IGF2, MLH1, NEUROG1, RUNX3, and SOCS1 [34]. The relative methylation level of long interspersed nucleotide element 1 (LINE-1) and IGF2 differentially methylated region 0 (DMR0) were quantified by pyrosequencing $[35,36]$.

Immunohistochemistry (IHC) analyses were conducted for the following protein markers: AURKA [37], CCND1 [38], CD274 (PD-L1) [39], CDH1 [40], CDK8 [41], CDKN1A [42], CDKN1B [42], CDKN2A [43], CDX2 [44], nuclear CTNNB1 [45], CTSB [46], DNMT3B [47], EPAS1 [48], FASN [49], HGF [50], HIF1A [48], IGF2BP3 [51], IRS1 [52], IRS2 [53], MGMT [54], PPARG [55], 
PTGER2 [56], PTGS2 [6], SIRT1 [57], STAT3 [58], TP53 [59], VDR [60], cytoplasmic YAP1 [61], and JC virus Tantigen (JCVT) [62]. For $\mathrm{T}$ cells in tumor tissue, we conducted IHC and image analysis on tissue microarray to measure densities of $\mathrm{CD} 3+, \mathrm{CD} 8+, \mathrm{CD} 45 \mathrm{RO}+$, and $\mathrm{FOXP}^{+}$cells $\left(\right.$cells $/ \mathrm{mm}^{2}$ ) [63]. Lymphocytic reactions were examined by the pathologist (S.O.) for tumorinfiltrating lymphocytes (i.e., lymphocytes on top of neoplastic epithelial cells, TIL), peritumoral lymphocytic reaction, intratumoral periglandular reaction, and Crohn's-like reaction [64].

RNA was extracted from the colorectal tumor tissue and adjacent non-tumor tissue within FFPE samples, and CDNA was synthesized as previously described [4]. The expression levels of MIR21 and MIR155 were quantified using miScript PCR System (Qiagen, Valencia, CA) with the StepOnePlus Real-Time PCR Systems (Applied Biosystems, San Diego, CA) [65].

\section{Statistical and network analysis}

To compare demographic characteristics of proximal colon cancer patients with distal colorectal cancer patients, a chi-squared test was used to compare two or more categorical variables by location, and a t-test was conducted to compare age by location.

In the correlation network analysis [66], a node represented a tumor tissue biomarker. The structure of correlations was captured by placing an edge between any two nodes whose associated biomarkers exhibited statistically significant Spearman correlation, where the significance level for correlations was chosen to be $3.5 \times 10^{-5}$ (= 0.05 / 1431, based on the Bonferroni correction). The pairwise correlation analysis was conducted as a complete case analysis in which patients without either biomarker information were excluded from the analysis. In the correlation analyses, we used continuous (for MSI and markers measuring levels of methylation, T-cell densities, miRNA expression, and Fusobacterium nucleatum), ordinal (for markers measuring tumor protein expression levels), and binary (for markers assessing mutation status) variables. The degree of a node was defined as the number of edges adjacent to the given node. We used a KolmogorovSmirnov (K-S) test to evaluate the distance between the cumulative degree distributions of the proximal colon cancer network and the distal colorectal cancer network. For each marker, degree centrality was computed as the fraction of nodes to which a node was connected (i.e., degree of a node divided by the number of all nodes in the network). A network node (marker) showing a high degree of connectedness, often referred to as a hub, is more likely to play an essential role in the disease network [14]. In the current study, hubs were defined as nodes with degree centrality greater than the 80th percentile based on overall colorectal cancer network that pooled both proximal colon and distal colorectal cancer. In each network, average clustering coefficient quantifies the overall clustering of nodes, indicating the tendency of markers to clustering together in the network [67]. For each node, a clustering coefficient was computed as the proportion of directly connected neighbors, and then an average of clustering coefficients was calculated in the network. To identify the markers that show distinct correlation patterns in proximal colon cancer versus distal colorectal cancer, we computed Cook's distance based on linear regression analysis [68]. In this analysis, the degree of each node in the distal colorectal cancer networks was regressed on the degree of each node in the proximal colon cancer networks.

To assess the robustness of our results from the network analysis, we conducted permutation tests and sensitivity analyses. First, to evaluate the null hypothesis of no difference in network edge counts by tumor location, we permutated the dataset 10,000 times by randomly assigning tumor location in 1380 patients. Each time, networks were constructed by tumor location, and the difference in the total number of edges was computed. The two-sided $P$ value was obtained as the proportion of random permutations that resulted in a difference in edge counts that was greater than or equal to the observed difference. Similarly, another permutation test was conducted by permuting values of each biomarker in 690 patients with proximal colon cancer and in 690 patients with distal colorectal cancer separately. In this process, we broke the linkage between each pair of biomarkers but retained imbalanced data (e.g., difference in mutation frequency) between tumor location. Second, across different significance levels of the Spearman correlation, we compared the number of edges in the proximal colon cancer network with that in the distal colorectal cancer network. Third, we used binary variables for all markers in the Spearman correlation analysis, and we constructed network models by tumor location. Fourth, we conducted a sensitivity analysis to include only selected markers that were available for more than $80 \%$ of the patients $(B R A F$, KRAS, PIK3CA, MLH1 methylation, CDKN2A methylation, CACNA1G methylation, CRABP1 methylation, IGF2 methylation, NEUROG1 methylation, RUNX3 methylation, SOCS1 methylation, MSI, LINE-1 methylation level, PTGS2, peritumoral lymphocytic reaction, intratumoral periglandular reaction, and TILs).

All the statistical analyses were carried out with SAS software (version 9.4, SAS Institute, Cary, NC). Network analysis was carried out with Python (version 2.7). All $P$ values were two-sided.

\section{Additional files}

Additional file 1: Figure S1. The biomarker network in colorectal cancer. A node represents a molecular feature, and an edge specifies the Spearman correlation between two markers with a significance level of 
$3.5 \times 10^{-5}(0.05 / 1431$, based on the Bonferroni correction). The red line indicates a positive correlation, and the blue line indicates a negative correlation. The line width is proportional to a correlation coefficient. CDKN2A (IHC), protein expression of CDKN2A; CDKN2A, methylation level of CDKN2A; LINE-1, methylation level of long interspersed nucleotide element 1; MSI, microsatellite instability; TIL, lymphocytes on top of neoplastic epithelial cells. (TIFF 344 KB)

Additional file 2: Figure S2. The biomarker networks in non-MSI-high colorectal cancer; proximal colon cancer network (A), and distal colorecta cancer network (B). (TIFF $325 \mathrm{~KB}$ )

Additional file 3: Figure S3. The biomarker networks with the same number of edges; proximal colon cancer network (A), and distal colorectal cancer network (B). (TIFF $311 \mathrm{~KB}$ )

Additional file 4: Table S1. Network characteristics by tumor location. (DOCX 16.4 KB)

\section{Abbreviations}

CIMP: CpG island methylator phenotype; FFPE: Formalin-fixed paraffinembedded; HPFS: Health Professionals Follow-up Study; IGF2 DMRO: IGF2 differentially methylated region 0; IHC: Immunohistochemistry; JCVT: JC Virus T-Antigen; K-S test: Kolmogorov-Smirnov test; MAPK: Mitogen-activated protein kinase; MSI: Microsatellite instability; MSS: Microsatellite instabilitylow/microsatellite stability; NHS: Nurses' Health Study; PCR: Quantitative polymerase chain reaction; SD: Standard deviation; TIL: Tumor infiltrating lymphocytes

\section{Acknowledgements}

We would like to thank the participants and staff of the Nurses' Health Study and the Health Professionals Follow-up Study for their valuable contributions as well as the following state cancer registries for their help: $A L, A Z, A R, C A$, $C O, C T, D E, F L, G A, I D, I L, I N, I A, K Y, L A, M E, M D, M A, M I, N E, N H, N J, N Y, N C$ ND, OH, OK, OR, PA, RI, SC, TN, TX, VA, WA, WY. The authors assume full responsibility for analyses and interpretation of these data.

\section{Funding}

This work was supported by U.S. National Institutes of Health $(\mathrm{NIH})$ grants [P01 CA87969 to M.J. Stampfer; UM1 CA186107 to MJ. Stampfer; P01 CA55075 to W.C. Willett; UM1 CA167552 to W.C. Willett; P50 CA127003 to C.S.F.; K24 DK098311 and R01 CA137178 to A.T.C.; R01 CA151993 to S.O.; R35 CA197735 to S.O.; and K07 CA190673 to R.N.]; Nodal Award (to S.O.) from the Dana-Farber Harvard Cancer Center; and by grants from the Project P Fund, The Friends of the Dana-Farber Cancer Institute, Bennett Family Fund, and the Entertainment Industry Foundation through National Colorectal Cancer Research Alliance. K.M. was supported by a grant from Program for Advancing Strategic International Networks to Accelerate the Circulation of Talented Researchers from Japanese Society for the Promotion of Science. T.H. was supported by a fellowship grant from the Uehara Memorial Foundation and by a grant from the Mochida Memorial Foundation for Medical and Pharmaceutical Research. The content is solely the responsibility of the authors and does not necessarily represent the official views of $\mathrm{NIH}$. The funders had no role in study design, data collection and analysis, decision to publish, or preparation of the manuscript.

\section{Availability of data and materials}

Data are available from the Harvard Cohorts Data Access Committee for researchers who meet the criteria for access to confidential data.

\section{Authors' contributions}

RN, SO, PK, JQ, and JPO contributed to the concept and design of the study. $R N, K M, T H, J A N$, and ZRQ preformed data collection and cleaning. RN and JPO conducted data analyses. RN, SO, and JPO drafted the manuscript. RN, KG, KM, TH, JAN, ZRQ, PK, ELG, CSF, ATC, JQ, SO, and JPO provided interpretation of data and critical revision of the manuscript. PK, ELG, CSF, ATC, and JQ provided supervision. All authors read and approved the final manuscript.

\section{Competing interests}

Dr. Chan was previously a consultant of Bayer Healthcare, Millennium Pharmaceuticals, and Pfizer Inc. This study was not funded by Bayer Healthcare, Millennium Pharmaceuticals, or Pfizer Inc. No other conflict of interest exists.

\section{Consent for publication}

Not applicable.

\section{Ethics approval and consent to participate}

Written informed consent was obtained from all participants. Deaths were ascertained through the National Death Index, postal authorities, and next of kin from whom permission was granted to review medical records. This study was approved by the Human Subjects Committees of Harvard T.H. Chan School of Public Health and Brigham and Women's Hospital.

\section{Publisher's Note}

Springer Nature remains neutral with regard to jurisdictional claims in published maps and institutional affiliations.

\section{Author details \\ ${ }^{1}$ Program of MPE Molecular Pathological Epidemiology, Department of Pathology, Brigham and Women's Hospital and Harvard Medical School, Boston, MA, USA. ²Department of Nutrition, Harvard T.H. Chan School of Public Health, Boston, MA, USA. ${ }^{3}$ Department of Biostatistics, Harvard T.H. Chan School of Public Health, Boston, MA, USA. ${ }^{4}$ Department of \\ Epidemiology, Harvard T.H. Chan School of Public Health, Boston, MA, USA. ${ }^{5}$ Channing Division of Network Medicine, Department of Medicine, Brigham and Women's Hospital and Harvard Medical School, Boston, MA, USA. ${ }^{6}$ Department of Medical Oncology, Dana-Farber Cancer Institute and Harvard Medical School, Boston, MA, USA. 7 Yale Cancer Center, New Haven, CT, USA. ${ }^{8}$ Department of Medicine, Yale School of Medicine, New Haven, CT, USA. ${ }^{9}$ Smilow Cancer Hospital, New Haven, CT, USA. ${ }^{10} \mathrm{Clinical}$ and Translational Epidemiology Unit, Massachusetts General Hospital and Harvard Medical School, Boston, MA, USA. ${ }^{11}$ Department of Biostatistics and Computational Biology, Dana-Farber Cancer Institute and Harvard Medical School, Boston, MA, USA. ${ }^{12}$ Department of Oncologic Pathology, Dana-Farber Cancer Institute and Harvard Medical School, Boston, MA, USA.}

Received: 13 February 2017 Accepted: 8 June 2017

Published online: 17 June 2017

\section{References}

1. Emmert-Streib F, de Matos SR, Glazko G, McDade S, Haibe-Kains B, Holzinger A, et al. Functional and genetic analysis of the colon cancer network. BMC Bioinformatics. 2014;15(Suppl 6):S6.

2. Vilar E, Gruber SB. Microsatellite instability in colorectal cancer-the stable evidence. Nat Rev Clin Oncol. 2010;7(3):153-62.

3. Weisenberger DJ, Levine AJ, Long TI, Buchanan DD, Walters R, Clendenning $\mathrm{M}$, et al. Association of the colorectal $\mathrm{CpG}$ island methylator phenotype with molecular features, risk factors, and family history. Cancer Epidemiol Biomark Prev. 2015;24(3):512-9.

4. Mima K, Nishihara R, Nowak JA, Kim SA, Song M, Inamura K, et al. MicroRNA MIR21 and T cells in colorectal cancer. Cancer Immunol Res. 2016;4(1):33-40.

5. Zhang GJ, Xiao HX, Tian HP, Liu ZL, Xia SS, Zhou T. Upregulation of microRNA-155 promotes the migration and invasion of colorectal cancer cells through the regulation of claudin-1 expression. Int J Mol Med. 2013;31(6):1375-80.

6. Mima K, Nishihara R, Yang J, Dou R, Masugi Y, Shi Y, et al. MicroRNA MIR2 (miR-21) and PTGS2 expression in colorectal cancer and patient survival. Clin Cancer Res. 2016;22(15):3841-8.

7. Pritchard CC, Grady WM. Colorectal cancer molecular biology moves into clinical practice. Gut. 2011;60(1):116-29.

8. Rozek LS, Schmit SL, Greenson JK, Tomsho LP, Rennert HS, Rennert G, Gruber SB. Tumor-Infiltrating Lymphocytes, Crohn's-Like Lymphoid Reaction, and Survival From Colorectal Cancer. J Natl Cancer Inst. 2016;108(8):djw027. doi:10.1093/jnci/djw027.

9. Hofree M, Shen JP, Carter H, Gross A, Ideker T. Network-based stratification of tumor mutations. Nat Methods. 2013;10(11):1108-15.

10. Mutation Consequences and Pathway Analysis working group of the International Cancer Genome Consortium. Pathway and network analysis of cancer genomes. Nat Methods. 2015;12(7):615-21.

11. Yamauchi M, Morikawa T, Kuchiba A, Imamura Y, Qian ZR, Nishihara R, et al. Assessment of colorectal cancer molecular features along bowel subsites challenges the conception of distinct dichotomy of proximal versus distal colorectum. Gut. 2012;61(6):847-54 
12. Missiaglia E, Jacobs B, D'Ario G, Di Narzo AF, Soneson C, Budinska E, et al. Distal and proximal colon cancers differ in terms of molecular, pathological, and clinical features. Ann Oncol. 2014;25(10):1995-2001.

13. Drewes JL, Housseau F, Sears CL. Sporadic colorectal cancer: microbial contributors to disease prevention, development and therapy. Br J Cancer. 2016;115(3):273-80.

14. Goh Kl, Cusick ME, Valle D, Childs B, Vidal M, Barabasi AL. The human disease network. Proc Natl Acad Sci U S A. 2007;104(21):8685-90.

15. Yang Y, Han L, Yuan Y, Li J, Hei N, Liang H. Gene co-expression network analysis reveals common system-level properties of prognostic genes across cancer types. Nat Commun. 2014;5:3231.

16. The Cancer Genome Atlas Network. Comprehensive molecular characterization of human colon and rectal cancer. Nature. 2012;487(7407):330-7.

17. Sadanandam A, Lyssiotis CA, Homicsko K, Collisson EA, Gibb WJ, Wullschleger $\mathrm{S}$, et al. A colorectal cancer classification system that associates cellular phenotype and responses to therapy. Nat Med. 2013;19(5):619-25.

18. Cahill DP, Kinzler KW, Vogelstein B, Lengauer C. Genetic instability and darwinian selection in tumours. Trends Cell Biol. 1999;9(12):M57-60.

19. Negrini S, Gorgoulis VG, Halazonetis TD. Genomic instability-an evolving hallmark of cancer. Nat Rev Mol Cell Biol. 2010;11(3):220-8.

20. Barras D. BRAF mutation in colorectal cancer: an update. Biomark Cancer. 2015;7(Suppl 1):9-12.

21. Phipps Al, Buchanan DD, Makar KW, Burnett-Hartman AN, Coghill AE, Passarelli MN, et al. BRAF mutation status and survival after colorectal cancer diagnosis according to patient and tumor characteristics. Cancer Epidemiol Biomark Prev. 2012;21(10):1792-8.

22. Zlobec I, Bihl M, Foerster A, Rufle A, Lugli A. Comprehensive analysis of CpG Island Methylator phenotype (CIMP)-high, -low, and -negative colorectal cancers based on protein marker expression and molecular features. J Pathol. 2011;225(3):336-43.

23. Gavin P, Colangelo LH, Fumagalli D, Tanaka N, Remillard MY, Yothers $G$, et al. Mutation profiling and microsatellite instability in stage II and III Colon cancer: an assessment of their prognostic and Oxaliplatin predictive value. Clin Cancer Res. 2012:18(23):6531-41.

24. Lochhead P, Kuchiba A, Imamura Y, Liao X, Yamauchi M, Nishihara R, et al. Microsatellite instability and BRAF mutation testing in colorectal cancer prognostication. J Natl Cancer Inst. 2013;105(15):1151-6.

25. Pai RK, Jayachandran P, Koong AC, Chang DT, Kwok S, Ma L, et al. BRAF-mutated, microsatellite-stable adenocarcinoma of the proximal colon: an aggressive adenocarcinoma with poor survival, mucinous differentiation, and adverse morphologic features. Am J Surg Pathol. 2012;36(5):744-52.

26. Imamura Y, Morikawa T, Liao X, Lochhead P, Kuchiba A, Yamauchi M, et al. Specific mutations in KRAS codons 12 and 13, and patient prognosis in 1075 BRAF wild-type colorectal cancers. Clin Cancer Res. 2012;18(17):4753-63.

27. Morkel M, Riemer P, Blaker $H$, Sers C. Similar but different: distinct roles for KRAS and BRAF oncogenes in colorectal cancer development and therapy resistance. Oncotarget. 2015;6(25):20785-800.

28. Dallol A, Buhmeida A, Al-Ahwal MS, Al-Maghrabi J, Bajouh O, Al-Khayyat S, et al. Clinical significance of frequent somatic mutations detected by highthroughput targeted sequencing in archived colorectal cancer samples. J Transl Med. 2016;14(1):118.

29. Pinheiro M, Pinto C, Peixoto A, Veiga I, Lopes P, Henrique R, et al. Target gene mutational pattern in lynch syndrome colorectal carcinomas according to tumour location and germline mutation. $\mathrm{Br} J$ Cancer. 2015:113(4):686-92.

30. Nishihara R, Lochhead P, Kuchiba A, Jung S, Yamauchi M, Liao X, et al. Aspirin use and risk of colorectal cancer according to BRAF mutation status. JAMA. 2013;309(24):2563-71.

31. Imamura Y, Lochhead P, Yamauchi M, Kuchiba A, Qian ZR, Liao X, et al. Analyses of clinicopathological, molecular, and prognostic associations of KRAS codon 61 and codon 146 mutations in colorectal cancer: cohort study and literature review. Mol Cancer. 2014;13:135.

32. Liao X, Morikawa T, Lochhead P, Imamura Y, Kuchiba A, Yamauchi M, et al. Prognostic role of PIK3CA mutation in colorectal cancer: cohort study and literature review. Clin Cancer Res. 2012;18(8):2257-68

33. Mima K, Nishihara R, Qian ZR, Cao Y, Sukawa Y, Nowak JA, Yang J, Dou R, Masugi $Y$, Song $M$, et al. Fusobacterium nucleatum in colorectal carcinoma tissue and patient prognosis. Gut. 2016;65:1973-80.

34. Ogino S, Kawasaki T, Kirkner GJ, Kraft P, Loda M, Fuchs CS. Evaluation of markers for $\mathrm{CpG}$ island methylator phenotype (CIMP) in colorectal cancer by a large population-based sample. J Mol Diagn. 2007;9(3):305-14
35. Irahara N, Nosho K, Baba Y, Shima K, Lindeman NI, Hazra A, et al. Precision of pyrosequencing assay to measure LINE-1 methylation in colon cancer, normal colonic mucosa, and peripheral blood cells. J Mol Diagn. 2010;12(2):177-83.

36. Baba Y, Nosho K, Shima K, Huttenhower C, Tanaka N, Hazra A, et al. Hypomethylation of the IGF2 DMR in colorectal tumors, detected by bisulfite pyrosequencing, is associated with poor prognosis. Gastroenterology. 2010;139(6):1855-64.

37. Baba Y, Nosho K, Shima K, Irahara N, Kure S, Toyoda S, et al. Aurora-a expression is independently associated with chromosomal instability in colorectal cancer. Neoplasia. 2009;11(5):418-25.

38. Ogino S, Nosho K, Irahara N, Kure S, Shima K, Baba Y, et al. A cohort study of cyclin D1 expression and prognosis in 602 colon cancer cases. Clin Cancer Res. 2009;15(13):4431-8.

39. Masugi Y, Nishihara R, Yang J, Mima $K$, da Silva A, Shi Y, Inamura K, Cao $Y$, Song M, Nowak JA, et al. Tumour CD274 (PD-L1) expression and T cells in colorectal cancer. Gut. 2016. doi:10.1136/gutjnl-2016-311421.

40. Kim SA, Inamura K, Yamauchi M, Nishihara R, Mima K, Sukawa Y, et al. Loss of $\mathrm{CDH} 1$ (E-cadherin) expression is associated with infiltrative tumour growth and lymph node metastasis. Br J Cancer. 2016;114(2):199-206.

41. Firestein R, Shima K, Nosho K, Irahara N, Baba Y, Bojarski E, et al. CDK8 expression in 470 colorectal cancers in relation to beta-catenin activation, other molecular alterations and patient survival. Int J Cancer. 2010; 126(12):2863-73.

42. Ogino S, Nosho K, Shima K, Baba Y, Irahara N, Kirkner GJ, et al. p21 expression in colon cancer and modifying effects of patient age and body mass index on prognosis. Cancer Epidemiol Biomark Prev. 2009;18(9):2513-21.

43. Shima K, Nosho K, Baba Y, Cantor M, Meyerhardt JA, Giovannucci EL, et al. Prognostic significance of CDKN2A ( $p 16$ ) promoter methylation and loss of expression in 902 colorectal cancers: cohort study and literature review. Int J Cancer. 2011:128(5):1080-94.

44. Baba Y, Nosho K, Shima K, Freed E, Irahara N, Philips J, et al. Relationship of CDX2 loss with molecular features and prognosis in colorectal cancer. Clin Cancer Res. 2009;15(14):4665-73.

45. Nan H, Morikawa T, Suuriniemi M, Imamura Y, Werner L, Kuchiba A, et al. Aspirin use, 8q24 single nucleotide polymorphism rs6983267, and colorectal cancer according to CTNNB1 alterations. J Natl Cancer Inst. 2013;105(24):1852-61

46. Chan AT, Baba Y, Shima K, Nosho K, Chung DC, Hung KE, et al. Cathepsin B expression and survival in colon cancer: implications for molecular detection of neoplasia. Cancer Epidemiol Biomark Prev. 2010; 19(11):2777-85.

47. Nosho K, Shima K, Irahara N, Kure S, Baba Y, Kirkner GJ, et al. DNMT3B expression might contribute to $\mathrm{CpG}$ island methylator phenotype in colorectal cancer. Clin Cancer Res. 2009:15(11):3663-71.

48. Baba Y, Nosho K, Shima K, Irahara N, Chan AT, Meyerhardt JA, et al. HIF1A overexpression is associated with poor prognosis in a cohort of 731 colorectal cancers. Am J Pathol. 2010;176(5):2292-301.

49. Ogino S, Nosho K, Meyerhardt JA, Kirkner GJ, Chan AT, Kawasaki T, et al. Cohort study of fatty acid synthase expression and patient survival in colon cancer. J Clin Oncol. 2008;26(35):5713-20.

50. Seneviratne D, Ma J, Tan X, Kwon YK, Muhammad E, Melhem M, et al. Genomic instability causes HGF gene activation in colon cancer cells, promoting their resistance to necroptosis. Gastroenterology. 2015;148(1): 181-91. e117

51. Lochhead P, Imamura Y, Morikawa T, Kuchiba A, Yamauchi M, Liao X, et al. Insulin-like growth factor 2 messenger RNA binding protein 3 (IGF2BP3) is a marker of unfavourable prognosis in colorectal cancer. Eur J Cancer. 2012;48(18):3405-13.

52. Hanyuda A, Kim SA, Martinez-Fernandez A, Qian ZR, Yamauchi M, Nishihara $R$, et al. Survival benefit of exercise differs by tumor IRS1 expression status in colorectal cancer. Ann Surg Oncol. 2016;23(3):908-17.

53. Day E, Poulogiannis G, McCaughan F, Mulholland S, Arends MJ, Ibrahim AE, et al. IRS2 is a candidate driver oncogene on $13 q 34$ in colorectal cancer. Int J Exp Pathol. 2013;94(3):203-11.

54. Shima K, Morikawa T, Baba Y, Nosho K, Suzuki M, Yamauchi M, et al. MGMT promoter methylation, loss of expression and prognosis in 855 colorectal cancers. Cancer Causes Control. 2011;22(2):301-9.

55. Ogino S, Shima K, Baba Y, Nosho K, Irahara N, Kure S, et al. Colorectal cancer expression of peroxisome proliferator-activated receptor gamma (PPARG, PPARgamma) is associated with good prognosis. Gastroenterology. 2009; 136(4):1242-50 
56. Baba Y, Nosho K, Shima K, Goessling W, Chan AT, Ng K, et al. PTGER2 overexpression in colorectal cancer is associated with microsatellite instability, independent of $\mathrm{CpG}$ island methylator phenotype. Cancer Epidemiol Biomark Prev. 2010;19(3):822-31.

57. Nosho K, Shima K, Irahara N, Kure S, Firestein R, Baba Y, et al. SIRT1 histone deacetylase expression is associated with microsatellite instability and $\mathrm{CpG}$ island methylator phenotype in colorectal cancer. Mod Pathol. 2009;22(7):922-32.

58. Morikawa T, Baba Y, Yamauchi M, Kuchiba A, Nosho K, Shima K, et al. STAT3 expression, molecular features, inflammation patterns, and prognosis in a database of 724 colorectal cancers. Clin Cancer Res. 2011;17(6):1452-62.

59. Morikawa T, Kuchiba A, Liao X, Imamura Y, Yamauchi M, Qian ZR, et al Tumor TP53 expression status, body mass index and prognosis in colorectal cancer. Int J Cancer. 2011;131(5):1169-78.

60. Kure S, Nosho K, Baba Y, Irahara N, Shima K, Ng K, et al. Vitamin D receptor expression is associated with PIK3CA and KRAS mutations in colorectal cancer. Cancer Epidemiol Biomark Prev. 2009;18(10):2765-72.

61. Barry ER, Morikawa T, Butler BL, Shrestha K, de la Rosa R, Yan KS, et al. Restriction of intestinal stem cell expansion and the regenerative response by YAP. Nature. 2013;493(7430):106-10.

62. Nosho K, Shima K, Kure S, Irahara N, Baba Y, Chen L, et al. JC virus T-antigen in colorectal cancer is associated with p53 expression and chromosomal instability, independent of CpG island methylator phenotype. Neoplasia. 2009:11(1):87-95.

63. Nosho K, Baba Y, Tanaka N, Shima K, Hayashi M, Meyerhardt JA, et al. Tumour-infiltrating T-cell subsets, molecular changes in colorectal cancer, and prognosis: cohort study and literature review. J Pathol. 2010;222(4):350-66.

64. Ogino S, Nosho K, Irahara N, Meyerhardt JA, Baba Y, Shima K, et al. Lymphocytic reaction to colorectal cancer is associated with longer survival, independent of lymph node count, microsatellite instability, and CpG island methylator phenotype. Clin Cancer Res. 2009;15(20):6412-20.

65. Mima K, Nishihara R, Yang J, Dou R, Masugi Y, Shi Y, da Silva A, Cao Y, Song M, Nowak J et al. MicroRNA <i>MIR21</i> (miR-21) and PTGS2 expression in colorectal cancer and patient survival. Clin Cancer Res. 2016;22(15):3841-48.

66. Barnett I, Onnela JP. Change point detection in correlation networks. Sci Rep. 2016;6:18893.

67. Watts DJ, Strogatz SH. Collective dynamics of 'small-world' networks. Nature. 1998;393(6684):440-2.

68. Cook DR. Influential observations in linear regression. J Am Stat Assoc. 1979;74(365):169-74

\section{Submit your next manuscript to BioMed Central and we will help you at every step:}

- We accept pre-submission inquiries

- Our selector tool helps you to find the most relevant journal

- We provide round the clock customer support

- Convenient online submission

- Thorough peer review

- Inclusion in PubMed and all major indexing services

- Maximum visibility for your research

Submit your manuscript at www.biomedcentral.com/submit

) Biomed Central 